\title{
Towards accurate positions of hydrogen atoms bonded to heavy metal atoms
}

\author{
M. Woińska, M. L. Chodkiewicz, S. Pawlędzio, K. Woźniak \\ Biological and Chemical Research Centre, Department of Chemistry, University of Warsaw, Żwirki $i$ Wigury 101, Warszawa 02-089, \\ Poland \\ mwoinska@chem.uw.edu.pl
}

Transition metal (TM) bound hydrides can be used as hydrogen storage materials, they also play the key role in processeses of catalysis, energy conversion and search for superconductvity. However, they are very difficult to study with the method of X-ray diffraction, on the one hand, due to the fact that hydrogen atom has only one electron with density is usually strongly shifted towards its bonding partner, on the other hand, because its weak diffraction signal is strongly screened by scattering from the electron-rich heavy metal. Moreover, it is difficult to collect high quality, let alone high resolution X-ray diffraction data for such compounds. The availability of neutron diffraction data, which are the a benchmark of hydrogen positions, is even more limited.

Recently, it was proven that Hirshfeld Atom Refinement (HAR), using aspherical atomic scattering factors, allows locating hydrogen atoms bonded to light elements based on standard resolution X-ray diffraction data with accuracy and precision very close to the one of neutron experiments [1]. This was a significant improvement compared to the most popular Independent Atom Model (IAM). Nevertheless, excluding this study, only 5 structures of complexes with TM-H bonds have been successfully refined with HAR [1,2] for only two of which a complementary neutron data set is available.

We present the results of HAR of around $11 \mathrm{X}$-ray structures of crystals of metaloorganic compounds containing hydrogen atoms bonded to heavy metals from period IV (Fe, Co, Cu, Ni), V (Nb, Ru, Rh, Sb) and VI (Os) for which also the corresponding neutron structure is available. Refinements were performed using Olex2 with application of atomic aspherical structure factors computed with the DiSCaMB library [3], based on Hirshfeld partition of molecular electron density calculated for the central molecule embeddded in a cluster of atomic charges and dipoles centered on the atomic nuclei of the surrounding molecules within the radius of $8 \AA$. Additionally, HARs without a cluster of multipoles were carried out with NoSpherA2 [2]. Wave functions were calculated using the DFT method, in each case B3LYP, $\mathrm{PBE}$ and M062X functionals were used. All the calculations were performed also in the version including relativistic effects as implemented in the DKH2 Hamiltonian approach. Various basis sets were tested.

Overall, the DiSCaMB-HAR procedure elongates the TM-H bond lengths, bringing them closer to the neutron values [4]. The level of improvement is dependent on the quality of the experimental data and the refinement. The most prominent example of a successful refinement is the structure of a metalloorganic complex containing a $\mathrm{Ru}-\mathrm{H}$ bond, which can be refined anisotropically to obtain the $\mathrm{Ru}-\mathrm{H}$ distance in very high agreement with the one in the neutron structure (neutron: 1.598(3) $\AA$, IAM: 1.56(2) $\AA$, DiSCaMBHAR(B3LYP/cc-pVTZ-DK): 1.593(11) A, DiSCaMB-HAR(PBE/cc-pVTZ-DK): 1.599(11) $\AA$ ). In four cases (Fe, Ru and Rh complexes), refinement of ADPs of hydrogen atoms was feasible. In the case of the Fe complex, HAR performed with SHADE2-estimated H ADPs was feasible and was used to evaluate the H APDs reifned with HAR. For two structures X-ray and neutron experiments were performed at the same temperature, thus a direct comparison of HAR and neutron isotropic temperature factors of hydrogen atoms will be presented. The data sets were ranked according to various parameters describing data quality and refinement quality both for the neutron and the X-ray data sets, which lead to the final joint neutron and X-ray data-refinement quality ranking. The ranking reflects well how favourable the HAR-neurton TM-H bond length comparison is and correlates with the number of electrons in the TM. Examples showing the influence of factors such as the position in the ranking and the method of obtaining the molecular wave function on the quality of TM-H and other X-H bond lengths obtained with $\mathrm{HAR}$ will be presented.

[1] Woińska, M., Grabowsky, S., Dominiak, P. M., Woźniak, K. \& Jayatilaka, D. (2016). Sci. Adv. 2, e1600192.

[2] F. Kleemiss, O. V. Dolomanov, M. Bodensteiner, N. Peyerimhoff, L. Midgley, L. J. Bourhis, A. Genoni, L. A. Malaspina, D. Jayatilaka, J. L. Spencer, F. White, B. Grundkötter-Stock, S. Steinhauer, D. Lentz, H. Puschmann, S. Grabowsky (2021). Chem. Sci. 12, $1675-1692$.

[3] Chodkiewicz, M. L., Migacz, S., Rudnicki, W., Makal, A., Kalinowski, J. A., Moriarty, N. W., Grosse-Kunstleve, R. W., Afonine, P. V., Adams, P. D. \& Dominiak, P. M. (2018). J. Appl. Cryst. 51, 193-199.

[4] Woińska, M., Chodkiewicz, M. L. and Woźniak, K., (2021). Chem. Commun., DOI: 10.1039/d0cc07661a, accepted.

Keywords: Hirshfeld Atom Refinement (HAR); transition metals; hydrogen positions; organometallic compounds; crystallographic software

This work was financially supported by the Polish National Science Centre (under OPUS grant: DEC-2018/31/B/ST4/02142). Computational facilities of Wroclaw Centre for Networking and Supercomputing of Wroclaw University of Technology and the PL-Grid infrastructure were used.

Acta Cryst. (2021), A77, C280 